\title{
Non-alcoholic steatohepatitis (NASH)
}

EASL-EASD-EASO

\section{Source}

. (2016). EASL-EASD-EASO Clinical Practice Guidelines for the management of nonalcoholic fatty liver disease Journal of Hepatology, vol. 64 (6), 1388-1402. doi:10.1016/j.jhep.2015.11.004

The diagnosis of NASH requires the joint presence of steatosis, ballooning and lobular inflammation. Other histological features can be seen in NASH but are not necessary for the diagnosis: portal inflammation, polymorphonuclear infiltrates, Mallory-Denk bodies, apoptotic bodies, clear vacuolated nuclei, microvacuolar steatosis and megamitochondria. Perisinusoidal fibrosis is also frequent, but not part of the diagnostic criteria. The term "borderline" NASH is confusing, unnecessary and should be abandoned. The prospectively designed FLIP algorithm increases observer agreement and precisely defines the grading of ballooning. "Burned-out NASH" describes regression of advanced disease (steatosis, inflammation or ballooning) in patients exposed to metabolic risk factors. The NAFLD Activity Score (NAS) scoring system should not be used for the diagnosis of NASH but rather for the evaluation of disease severity once the diagnosis has been established by the overall pathological assessment. In children, NASH displays many of the features observed in adults, even though the distribution of lesions may be different. Portal inflammation is a frequent feature but can also be seen in adults with more severe disease. Hepatocellular ballooning and Mallory-Denk bodies are only sporadically observed in paediatric NASH, and portal-based chronic inflammation is predominant. 